CERN-EP/99-90

July 5, 1999

\title{
MEASUREMENT OF THE SMC MUON BEAM POLARISATION USING THE ASYMMETRY IN THE ELASTIC SCATTERING OFF POLARISED ELECTRONS
}

The Spin Muon Collaboration (SMC)

\begin{abstract}
A muon beam polarimeter was built for the SMC experiment at the CERN SPS, for beam energies of 100 and $190 \mathrm{GeV}$. The beam polarisation is determined from the asymmetry in the elastic scattering off the polarised electrons of a ferromagnetic target whose magnetisation is periodically reversed. At muon energies of 100 and $190 \mathrm{GeV}$ the measured polarisation is $P_{\mu}=-0.80 \pm 0.03$ (stat.) \pm 0.02 (syst.) and $P_{\mu}=-0.797 \pm 0.011$ (stat.) \pm 0.012 (syst.), respectively. These results agree with measurements of the beam polarisation using a shape analysis of the decay positron energy spectrum.
\end{abstract}

Submitted to Nuclear Instruments and Methods 
D. Adams ${ }^{17)}$, B. Adeva ${ }^{19)}$, T. Akdogan ${ }^{2)}$, E. Arik ${ }^{2)}$, A. Arvidson $\left.22, a\right), B . B^{2} \operatorname{Bdelek}^{22,24)}$, G. Bardin ${ }^{18, \text { ee) }}$, G. Baum ${ }^{1)}$, P. Berglund ${ }^{8)}$, L. Betev ${ }^{13)}$, R. Birsa ${ }^{21)}$, P. Björkholm ${ }^{22, a a)}$, B.E. Bonner ${ }^{17)}$, N. de Botton ${ }^{18)}$, M. Boutemeur ${ }^{25, c c)}$, F. Bradamante ${ }^{21)}$, A. Bravar ${ }^{11)}$, A. Bressan ${ }^{21, n)}$, S. Bültmann ${ }^{1, b)}$, E. Burtin ${ }^{18)}$, C. Cavata ${ }^{18)}$, M. Clocchiatti ${ }^{21)}$, D. Crabb ${ }^{23)}$, J. Cranshaw ${ }^{21)}$, T. Çuhadar ${ }^{2,15)}$, S. Dalla Torre ${ }^{21)}$, R. van Dantzig ${ }^{15)}$,

B. Derro ${ }^{4)}$, A. Deshpande ${ }^{25)}$, S. Dhawan ${ }^{25)}$, C. Dulya ${ }^{15,4, \mathrm{c})}$, A. Dyring ${ }^{22)}$, S. Eichblatt ${ }^{17,,)}$ J.C. Faivre ${ }^{18)}$, D. Fasching ${ }^{16, e)}$, F. Feinstein ${ }^{18)}$, C. Fernandez ${ }^{19,9)}$, S. Forthmann ${ }^{7)}$, B. Frois ${ }^{18)}$, A. Gallas ${ }^{19)}$, J.A. Garzon ${ }^{19,9)}$, L. Gatignon ${ }^{5)}$, T. Gaussiran ${ }^{21)}$, H. Gilly ${ }^{6}$, M. Giorgi ${ }^{21)}$, E. von Goeler ${ }^{\text {) }}$, S. Goertz ${ }^{3)}$, I. A. Golutvin ${ }^{10)}$, G. Gracia ${ }^{19, f)}$, N. de Groot ${ }^{15, \mathrm{~g})}$, M. Grosse Perdekamp ${ }^{25, \mathrm{w})}$, K. Haft ${ }^{13)}$, D. von Harrach ${ }^{11)}$, T. Hasegawa ${ }^{14, \mathrm{~h})}$, P. Hautle ${ }^{5, \mathrm{i})}$, N. Hayashi ${ }^{14, \mathrm{j})}$, C.A. Heusch $^{5, \mathrm{k})}$, N. Horikawa ${ }^{14)}$, V.W. Hughes ${ }^{25)}$, G. Igo $^{4)}$, S. Ishimoto ${ }^{14,1)}$, T. Iwata ${ }^{14)}$, E.M. Kabuß ${ }^{11)}$, T. Kageya ${ }^{14, \mathrm{~m})}$, A. Karev ${ }^{10)}$, H.J. Kessler ${ }^{6, v)}$, T.J. Ketel ${ }^{15)}$, J. Kiryluk ${ }^{24)}$, I. Kiryushin ${ }^{10)}$, A. Kishi ${ }^{14)}$, Yu. Kisselev ${ }^{10)}$, L. Klostermann ${ }^{15)}$ D. Krämer ${ }^{1)}$, V. Krivokhijine ${ }^{10)}$, W. Kröger ${ }^{5, k)}$, V. Kukhtin ${ }^{10)}$, K. Kurek ${ }^{24)}$, J. Kyynäräinen ${ }^{1,8)}$, M. Lamanna ${ }^{21, n)}$, U. Landgraf ${ }^{6)}$, J.M. Le Goff ${ }^{18)}$, F. Lehar ${ }^{18)}$, A. de Lesquen ${ }^{18)}$, J. Lichtenstadt ${ }^{20)}$, T. Lindqvist ${ }^{22)}$, M. Litmaath ${ }^{15, n)}$, M. Lowe ${ }^{17)}$, A. Magnon ${ }^{18)}$, G.K. Mallot ${ }^{11, n)}$, F. Marie ${ }^{18)}$, A. Martin ${ }^{21)}$, J. Martino ${ }^{18)}$, T. Matsuda ${ }^{14, h)}$, B. Mayes ${ }^{9)}$, J.S. McCarthy ${ }^{23)}$, K. Medved ${ }^{10)}$, W. Meyer ${ }^{3)}$, G. van Middelkoop ${ }^{15)}$, D. Miller ${ }^{16)}$, Y. Miyachi ${ }^{14)}$, K. Mori $^{14)}$, J. Moromisato ${ }^{\text {) }}$,

A. Nagaitsev ${ }^{10)}$, J. Nassalski ${ }^{24)}$, L. Naumann ${ }^{5, \mathrm{ee})}$, T.O. Niinikoski ${ }^{5)}$, J.E.J. Oberski ${ }^{15)}$, A. Ogawa ${ }^{14, \mathrm{p})}$, C. Ozben $^{2)}$, H. Pereira ${ }^{18)}$, F. Perrot-Kunne ${ }^{18)}$, D. Peshekhonov ${ }^{10)}$,

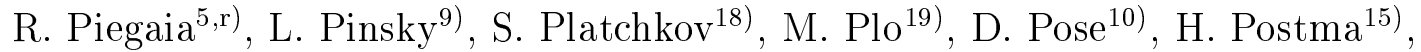
J. Pretz ${ }^{11, \mathrm{x})}$, T. Pussieux ${ }^{18)}$, G. Rädel ${ }^{5)}$, A. Rijllart ${ }^{5)}$, G. Reicherz ${ }^{3)}$, J.B. Roberts ${ }^{17)}$, S. Rock ${ }^{5, y)}$, M. Rodriguez ${ }^{22, r)}$, E. Rondio ${ }^{24,5)}$, L. Ropelewski ${ }^{24, n)}$, I. Sabo ${ }^{20)}$, J. Saborido ${ }^{19, \mathrm{n})}$, A. Sandacz ${ }^{24)}$, I. Savin ${ }^{10)}$, P. Schiavon ${ }^{21)}$, A. Schiller ${ }^{7)}$,

K. P. Schüler ${ }^{25, d d)}$, R. Seitz ${ }^{11, b b)}$, Y. Semertzidis ${ }^{5, z)}$, S. Sergeev ${ }^{10)}$, P. Shanahan ${ }^{16, d)}$ E. P. Sichtermann ${ }^{15)}$, F. Simeoni ${ }^{21)}$, G.I. Smirnov ${ }^{10)}$, A. Staude ${ }^{13)}$, A. Steinmetz ${ }^{11,13)}$, U. Stiegler ${ }^{5)}$, H. Stuhrmann ${ }^{7)}$, M. Szleper ${ }^{24)}$, F. Tessarotto ${ }^{21)}$, D. Thers ${ }^{18)}$,

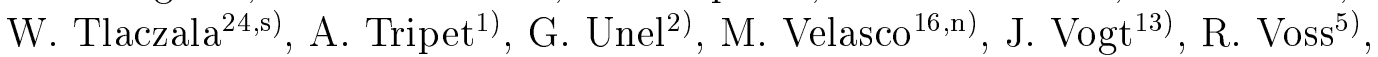
C. Whitten ${ }^{4)}$, R. Windmolders ${ }^{12)}$, R. Willumeit ${ }^{7)}$, W. Wislicki ${ }^{24)}$, A. Witzmann ${ }^{6, t)}$, J. Ylöstalo ${ }^{8)}$, A.M. Zanetti ${ }^{21)}$, K. Zaremba ${ }^{24, \mathrm{~s})}$, N. I. Zamiatin ${ }^{10)}$, J. Zhao ${ }^{7, \mathrm{u})}$ 
1) University of Bielefeld, Physics Department, 33501 Bielefeld, Germany

2) Bogaziçi University and Istanbul Technical University, Istanbul, Turkey

3) University of Bochum, Physics Department, 44780 Bochum, Germany

4) University of California, Department of Physics, Los Angeles, 90024 CA, USA

5) CERN, 1211 Geneva 23, Switzerland

6) University of Freiburg, Physics Department, 79104 Freiburg, Germany

7) GKSS, 21494 Geesthacht, Germany

8) Helsinki University of Technology, Low Temperature Laboratory and Institute of Particle Physics Technology, Espoo, Finland

9) University of Houston, Department of Physics, Houston, 77204-5506 TX, USA

10) JINR, Dubna, RU-141980 Dubna, Russia

11) University of Mainz, Institute for Nuclear Physics, 55099 Mainz, Germany

12) University of Mons, Faculty of Science, 7000 Mons, Belgium

13) University of Munich, Physics Department, 80799 Munich, Germany

14) Nagoya University, CIRSE and Department of Physics, Furo-Cho, Chikusa-Ku, 464 Nagoya, Japan

15) NIKHEF, Delft University of Technology, FOM and Free University, 1009 AJ Amsterdam, The Netherlands

16) Northwestern University, Department of Physics, Evanston, 60208 IL, USA

17) Rice University, Bonner Laboratory, Houston, 77251-1892 TX, USA ${ }^{\text {ccc })}$

18) C.E.A. Saclay, DAPNIA, 91191 Gif-sur-Yvette, France

19) University of Santiago, Department of Particle Physics, 15706 Santiago de Compostela, Spain

20) Tel Aviv University, School of Physics, 69978 Tel Aviv, Israel

21) INFN Trieste and University of Trieste, Department of Physics, 34127 Trieste, Italy

22) Uppsala University, Department of Radiation Sciences, 75121 Uppsala, Sweden

23) University of Virginia, Department of Physics, Charlottesville, 22901 VA, USA

24) Soltan Institute for Nuclear Studies and Warsaw University, 00681 Warsaw, Poland

25) Yale University, Department of Physics, New Haven, 06511 CT, USA

a) Now at The Royal Library, 10241 Stockholm, Sweden

b) Now at University of Virginia, Department of Physics, Charlottesville, 22901 VA, USA

c) Now at CIEMAT, Avda Complutense 22, 28040, Madrid, Spain

d) Now at Fermi National Accelerator Laboratory, Batavia, 60510 IL, USA

e) Now at University of Wisconsin, USA

f) Now at NIKHEF P.O.B. 41882, 1009 DB Amsterdam, The Netherlands

g) Now at Bristol University, Bristol, UK

h) Permanent address: Miyazaki University, Faculty of Engineering, 889-21 Miyazaki-Shi, Japan

i) Permanent address: Paul Scherrer Institut, 5232 Villigen, Switzerland

j) Permanent address: The Institute of Physical and Chemical Research (RIKEN), Wako 351-01, Japan

k) Permanent address: University of California, Institute of Particle Physics, Santa Cruz, 95064 CA, USA

1) Permanent address: KEK, Tsukuba-Shi, 305 Ibaraki-Ken,Japan

m) Now at University of Michigan, Ann Arbor MI48109, USA

n) Now at CERN, 1211 Geneva 23, Switzerland

o) Permanent address: Northeastern University, Department of Physics, Boston, 02115 MA, USA

p) Now at Penn. State University, 303 Osmond Lab, University Park, 16802 PA, USA

r) Permanent address: University of Buenos Aires, Physics Department, 1428 Buenos Aires, Argentina

s) Permanent address: Warsaw University of Technology, Warsaw, Poland 


\section{INTRODUCTION}

Experiments on deep inelastic scattering of polarised leptons on polarised nucleons are intended to measure the spin structure functions of the nucleon. Muon beams present the advantage of higher energies than those for available electron beams. This allows the extension of the kinematical range to lower values of Bjorken $x$, which are of importance for checking sum rules. Indeed, the EMC experiment [1] using the CERN SPS muon beam of $92-280 \mathrm{GeV}$ concluded for the first time on the violation of the Ellis-Jaffe sum rule by extending the measurement of the proton spin structure function $g_{1}^{p}(x)$ down to $x=0.01$. The EMC muon beam polarisation was determined using a Monte Carlo simulation of the beam phase space from the hadron production target to the experiment; the quoted relative uncertainty was $7.5 \%$. On the other hand, high energy electron polarimeters reach accuracies of a few percent using asymmetry measurements either in polarised Moller scattering [2] [3] or in polarised Compton laser backscattering [4] [5] .

The first experimental attempt to determine the polarisation of a muon beam was intended to check the weak interaction theory prediction of $a+1 / 2$ helicity value for the muon arising from the decay of a negative pion [6]. It consisted in the measurement of the asymmetry in the production of knock-on electrons on a series of iron foils whose magnetisation was periodically reversed. The electrons were identified and their energy was measured in a shower detector consisting of plastic scintillators interleaved with the iron foils. The helicity of the muon was shown to be compatible with $+1 / 2$ at a $30 \%$ accuracy level. Clearly the exploratory character of this early attempt left room for substantial improvements by a dedicated set-up which would perform a more complete identification of the same polarisation dependent process.

The SMC experiment which measured the spin structure function of the proton with an improved accuracy [7], and for the first time the spin structure of the deuteron [8], has developed a polarimeter aiming at the determination of the CERN SPS muon beam polarisation with a relative accuracy of a few percent. Two methods were implemented to achieve this goal. The first one derives the value of the polarisation from the analysis of the shape of the energy spectrum of the muon decay positrons [9] [10]. In this paper we will describe the second method which measures the asymmetry in polarised elastic $\mu$-e scattering [11][12].

\section{POLARISED MUON ELECTRON ELASTIC SCATTERING \\ 2.1 Kinematics}

The kinematics of muon electron elastic scattering

$$
\mu(k)+e(p) \rightarrow \mu\left(k^{\prime}\right)+e\left(p^{\prime}\right)
$$

\footnotetext{
t) Now at F.Hoffmann-La Roche Ltd., CH-4070 Basel, Switzerland

u) Now at Los Alamos National Laboratory, Los Alamos, NM 87545, USA

v) Now at SBC Warburg Dillon Read, CH-4002 Basel, Switzerland

w) Now at University of Mainz, Institute of Nuclear Physics, 55099, Germany

x) Now at Physics Department, Yale University, New Haven CT 06520, USA

y) Permanent address: The American University, Washington D.C. 20016, USA

z) Permanent address: Brookhaven National Laboratory, Upton, 11973, NY, USA

aa) Now at Ericsson Infotech AB, Karlstad, Sweden

bb) Now at Université de Montréal, Montréal, PQ, H3C 3J7,Canada

cc) Now at University of Munich, Physics Department, 80799 Munich, Germany

dd) Now at DESY, Notkestrasse 85, Hamburg, Germany

ee) Deceased.
} 
involve three independent variables. In the laboratory frame, where the target electron is at rest, we can choose them to be the incident muon energy $E_{\mu}$, the azimuthal angle $\phi$ of the scattering plane, and the fractional energy loss of the muon $y=1-E_{\mu}^{\prime} / E_{\mu}=E_{e}^{\prime} / E_{\mu}$, where $E_{e}^{\prime}$ and $E_{\mu}^{\prime}$ are the recoil electron and the scattered muon energy, respectively. Useful additional kinematical quantities are the square of the center of mass energy $s=m_{\mu}^{2}+m_{e}^{2}+2 m_{e} E_{\mu}, S=s-m_{e}^{2}-m_{\mu}^{2}$, and $\lambda_{S}=S^{2}-4 m_{e}^{2} m_{\mu}^{2}$.

Owing to the larger mass of the muon there is a maximum value for the energy which can be transferred to the electron and the corresponding muon fractional energy loss $Y$ is given by

$$
Y=\frac{\lambda_{S}}{s S} \simeq \frac{1}{1+m_{\mu}^{2} / 2 m_{e} E_{\mu}} .
$$

The angles $\theta_{\mu}$ of the scattered muon, and $\theta_{e}$ of the recoil electron in the laboratory frame are given by

$$
\theta_{\mu}=\sqrt{\frac{2 m_{e}}{E_{\mu}} \frac{y}{1-y}-\left(\frac{m_{\mu}}{E_{\mu}} \frac{y}{1-y}\right)^{2}} \text { and } \theta_{e}=\frac{y}{1-y} \theta_{\mu} .
$$

The maximum muon scattering angle is $\theta_{\mu}^{\max }=m_{e} / m_{\mu}=0.0048 \mathrm{rad}$.

\subsection{First order cross section}

The cross section in the laboratory frame for polarised elastic muon electron scattering in first order QED has been calculated [13] using the ultrarelativistic approximation for the electron, but taking into account the finite muon mass. In the usual coordinate system

$$
\mathbf{e}_{\mathrm{z}}=\mathbf{k} /|\mathbf{k}|, \quad \mathbf{e}_{\mathbf{y}}=\frac{\mathbf{k} \times \mathbf{k}^{\prime}}{\left|\mathbf{k} \times \mathbf{k}^{\prime}\right|}, \quad \mathbf{e}_{\mathbf{x}}=\mathbf{e}_{\mathbf{y}} \times \mathbf{e}_{\mathbf{z}},
$$

for polarisations $P_{e}$ and $P_{\mu}$ of the target electron and of the incoming positive muon, it reads :

$$
\frac{d^{2} \sigma}{d y d \phi}=\frac{d^{2} \sigma_{0}}{d y d \phi}\left(1+\sum a_{i j} P_{e}^{i} P_{\mu}^{j}\right)
$$

where the non polarised Born cross section is :

$$
\frac{d^{2} \sigma_{0}}{d y d \phi}=\frac{2 \alpha^{2} S}{\lambda_{S}}\left(\frac{1}{y^{2}}-\frac{s}{y S}+\frac{1}{2}\right)
$$

and the asymmetries are :

$$
\begin{gathered}
a_{z z}=\frac{\frac{-1}{y}+\frac{1}{Y}-\frac{1}{2}}{\frac{1}{y^{2}}-\frac{s}{y S}+\frac{1}{2}}, \quad a_{z x}=\frac{\frac{\hat{y} m_{\mu} \sqrt{S}}{y \sqrt{\lambda_{S}}}\left(1+\frac{2 m_{e}^{2}}{S}\right)}{\frac{1}{y^{2}}-\frac{s}{y S}+\frac{1}{2}}, \\
a_{x z}=\frac{\frac{\hat{y} m_{e} \sqrt{S}}{y \sqrt{\lambda_{S}}}\left(1+\frac{2 m_{\mu}^{2}}{S}\right)}{\frac{1}{y^{2}}-\frac{s}{y S}+\frac{1}{2}}, \quad a_{x x}=\frac{\frac{-2 m_{e} m_{\mu}}{y S}\left(2-\frac{y}{Y}\right)}{\frac{1}{y^{2}}-\frac{s}{y S}+\frac{1}{2}}, \quad a_{y y}=\frac{\frac{2 m_{e} m_{\mu}}{y S}}{\frac{1}{y^{2}}-\frac{s}{y S}+\frac{1}{2}},
\end{gathered}
$$

where $\hat{y}=\sqrt{y\left(1-\frac{y}{Y}\right)}$.

Because of parity and time reversal symmetry, $a_{x y}=a_{y x}=a_{z y}=a_{y z}=0$. 


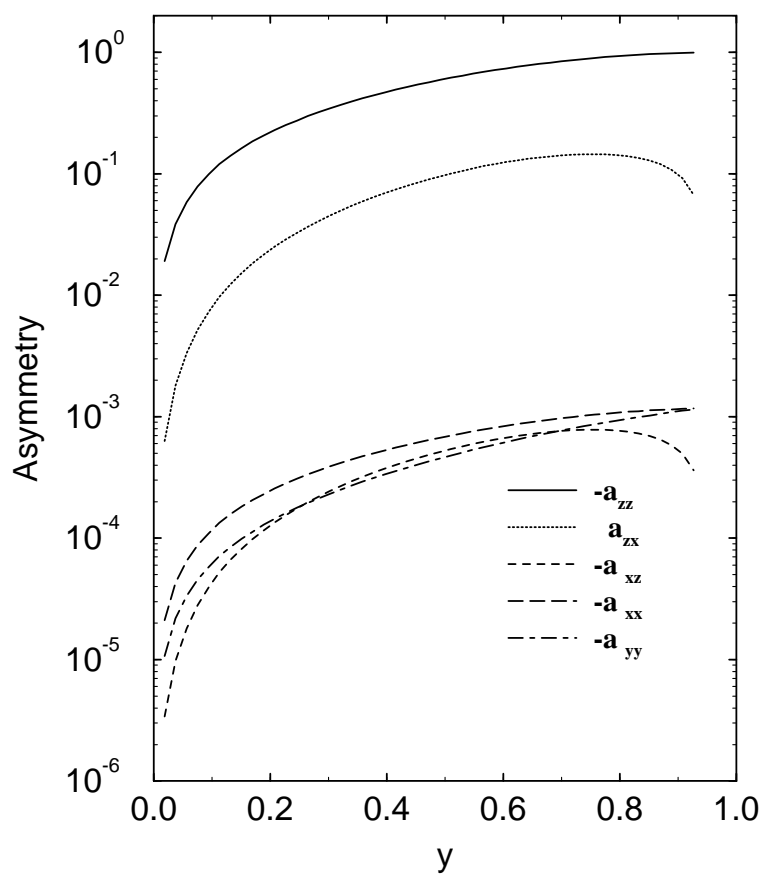

Figure 1: Born asymmetries of the $\mu^{+}-e^{-}$elastic scattering reaction for the incoming muon energy $E_{\mu}=190 \mathrm{GeV}$

The Born cross-section asymmetries are presented in Fig. 1. The only significant ones are $a_{z z}$ and $a_{z x}$. In our measurement in which polarisations are mostly longitudinal the asymmetry $a_{z z}$ is dominant ; we will label it $A$, and we will label the longitudinal polarisations of the muon beam and of the target electron $P_{\mu}$ and $P_{e}$, respectively. The contribution of the transverse asymmetry $a_{z x}$ brings only a small correction.

A relevant quantity for our asymmetry measurement is the figure of merit $A \sqrt{d^{2} \sigma / d y d \phi}$ which is inversely proportional to the statistical accuracy assuming a constant efficiency. For $E_{\mu}=190 \mathrm{GeV}$ it decreases monotonically by $30 \%$ from $y=0$ to $y=Y$.

\subsection{Radiative corrections}

The Feynman diagrams for the first order radiative corrections to elastic muon electron scattering are shown in Fig. 2. Diagrams $b, c, e$ and $f$ are for bremsstrahlung : they do not correspond to the elastic scattering reaction, but their kinematics are not experimentally distinguishable from the elastic channel. The bremsstrahlung terms contribute directly to the order $\alpha^{3}$. The other terms, of order $\alpha^{4}$, contribute through their interference with the Born term : $a$ and $d$ represent the vertex renormalisation, $g$ and $h$ the exchange of two photons, and $i$ the vacuum polarisation. Four gauge-invariant contributions to the radiative corrections can be calculated from the diagrams of Fig. 2 : a muonic term, an electronic term, a term originating in the interference of muonic and electronic terms, and the vacuum polarisation term. These four contributions are shown in Figs.3(a)-(d).

A program has been written [13] to calculate the radiative corrections reproducing the SMC polarimeter experimental conditions, and the cuts applied at the analysis level

- on the minimum recoil electron energy

- on the missing energy in the final state : $E_{\mu}-E_{\mu}^{\prime}-E_{e}^{\prime}$

- on the difference between the measured scattering angle and its value calculated from the measured value of the scattered muon momentum both for the muon and 


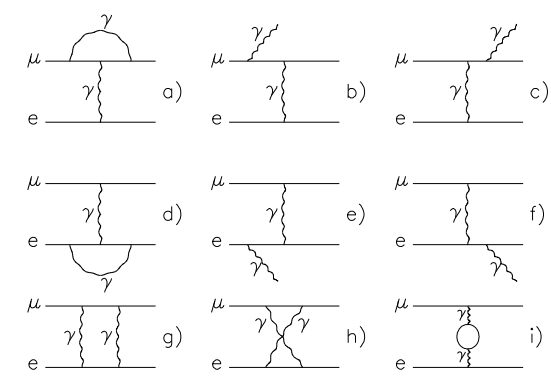

Figure 2: Feynmann diagrams for radiative corrections to $\mu-e$ scattering.

for the electron (see Eq. 2).

A detailed discussion of these cuts can be found in Sec. 4.3 .

The cuts reject part of the bremsstrahlung events. Fig. 3 shows the contributions from the four gauge invariant contributions and the total radiative corrections to the longitudinal asymmetry ; they amount to less than $0.5 \%$ throughout the $y$ range we have considered in our measurement.

\subsection{Sources of background}

Along with $\mu-e$ scattering there are two competing sources for the production of electrons by muons : muon bremsstrahlung $\left(\alpha^{3}\right)$ on a nucleus followed by photon conversion on another nucleus and muon direct $e^{+} e^{-}$pair production $\left(\alpha^{4}\right)$, where only one nucleus is involved. Both processes are primarily interactions with nuclei, and therefore they are independent of the target electron polarisation and produce a dilution of the asymmetry. The relative importance of these processes depends on target thickness. For the target thicknesses used in this experiment (see Table 2) bremsstrahlung predominates over direct pair production.

These background processes differ kinematically from $\mu-e$ scattering in two important respects: energy conservation in the observed final state, and scattering angles. Since we only measure the $\mu^{+}$and the $e^{-}$energies, these background events will always be missing the energy carried by the undetected $e^{+}$. The electron from a $\mu-e$ scattering event is degraded ; this produces a tail in the missing energy distribution. A loose cut on this tail rejects mostly background.

The angles differ because the final state for $\mu-e$ scattering events obeys 2-body kinematics with a well defined correlation between angles and momenta, whereas the background events follow 3-body kinematics with an angular distribution strongly peaked in the forward direction.

The characteristic angle of emission for muon bremsstrahlung is $m_{\mu} / E_{\mu}$ which is around $0.5 \mathrm{mrad}$ at $190 \mathrm{GeV}$. The conversion pair deviates from this direction only at the level of $m_{e} /\left(y E_{\mu}\right) \leq 25 \mu \mathrm{rad}$. The difference in the angle for bremsstrahlung and elastic scattering both for the produced electron and for the scattered muon are presented in Fig. 4, as well as the cuts used in the analysis to reject background. 

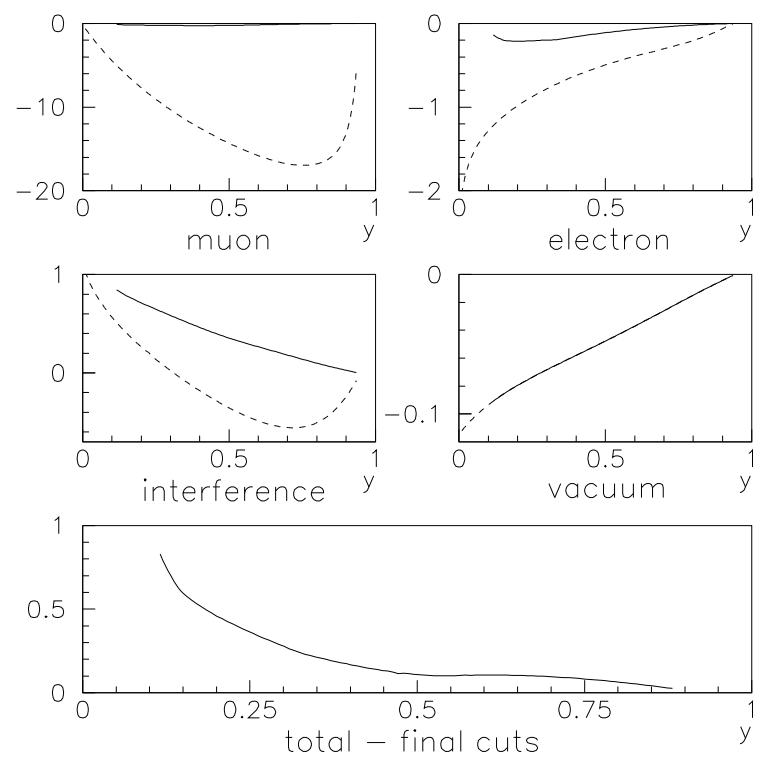

Figure 3: The radiative corrections $\left(A_{\mathrm{corr}}-A_{\mathrm{Born}}\right) / A_{\mathrm{Born}}$ (in percent) to the asymmetry for elastic electron-muon scattering as a function of $y$. The muonic, electronic, interference and vacuum contributions are shown both for no cut applied (dashed line) and for the final set of cuts described in the text (solid line). The dashed and solid lines are superimposed for the vacuum contribution as it is not affected by kinematical cuts. The last plot shows the sum of the previous four contributions for the final cuts.

Taking advantage of the charge symmetry property of electromagnetic interactions, a measurement of radiative background was performed using a $\mu^{-}$beam and requiring a coincidence of the scattered muon with an $e^{+}$in the electron arm of the spectrometer whose magnetic field was inverted.

The radiative background was also studied with a Monte Carlo simulation in which the bremsstrahlung angular spectrum was approximated by the distribution $\left(\gamma \theta_{\text {photon }}\right) /\left(1+\left(\gamma \theta_{\text {photon }}\right)^{2}\right)^{2}$, where $\gamma=E_{\mu} / m_{\mu}$, and $\theta_{\text {photon }}$ is the angle between the emitted photon and the incoming muon.

\section{EXPERIMENTAL SET UP}

The experimental method consists in the measurement of the asymmetry for the elastic scattering of the muons off the polarised electrons of a magnetised target whose magnetisation is inverted between beam pulses[14]. The incoming muon, the scattered muon and the recoil electron are identified and measured in a spectrometer which consists of a dipole magnet, several planes of scintillators and multi-wire proportional chambers, and a lead glass shower detector.

\subsection{The muon beam}

The CERN M2 muon beam [15] is produced from the decay of secondary hadrons (pions and kaons) generated by bombarding a beryllium target with the $450 \mathrm{GeV}$ SPS proton beam. The beam intensity is typically $4 \cdot 10^{7} \mu^{+}$per $2.4 \mathrm{~s}$ pulse, with a repetition rate of 250 pulses per hour. The typical transverse size ( $\mathrm{rms}$ ) of the the muon beam at the 


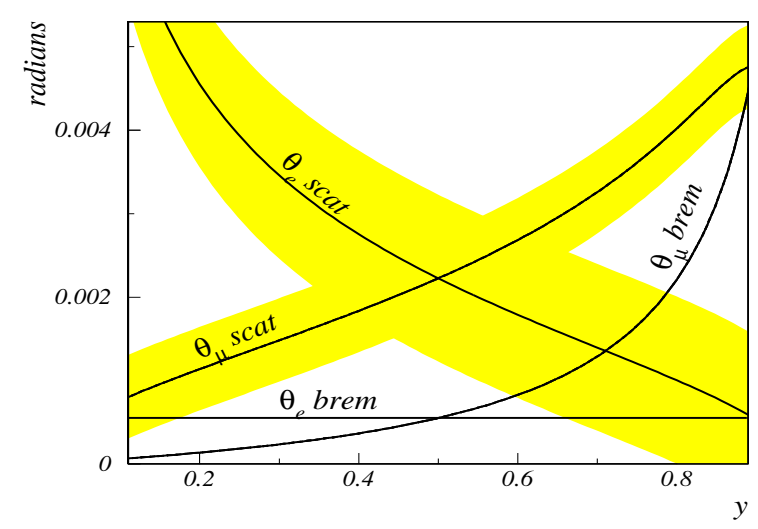

Figure 4: Scattering angles for $\mu-e$ scattering, and bremsstrahlung events. The lines for bremsstrahlung are for the characteristic angle. The shaded areas around the scattering angles indicate cuts used in the analysis described in Sec. 4.3

polarimeter is $25 \mathrm{~mm}$. Each muon is tagged by its momentum which is determined with a relative resolution of $3 \cdot 10^{-3}$ in the Beam Momentum Station (BMS) using four scintillator hodoscopes located upstream and downstream of an analysing magnetic system upstream of the SMC experimental hall [16].

For a given muon energy the muon polarisation is a decreasing function of the energy of the hadron parent. The muon beam energy spectrum is measured by the BMS. The hadron beam energy spectrum depends on the aperture of the momentum slits of the analysing magnet which define the useful hadron beam upstream of the hadron decay channel. The slit aperture during our measurement corresponded to a relative hadron energy range $\pm 4 \%$. Correction to the muon beam polarisation for a different slit aperture was estimated using a Monte Carlo simulation of the beam line.

The decay section and the muon beam transport system which consist of structures of alternately focussing and defocussing quadrupoles have an azimuthal symmetry; therefore the beam polarisation at the output is purely longitudinal. A small transverse polarisation is induced by the muon spin precession [17] during deflections by the dipole magnets of the beam transport system to the experimental hall. At the magnetised target location, the horizontal and vertical angles between the polarisation and the momentum of the muon were respectively 0.017 and 0.020 radian for $190 \mathrm{GeV}$ muons. Because of our experimental procedure (see Sec. 4.1) which averages over two target configurations with symmetrical angles with respect to the beam direction, the contribution of transverse target polarisation cancels out. Only the beam transverse polarisation had a small contribution to the measured asymmetry through the $a_{x z}$ asymmetry.

\subsection{The polarised electron target}

In order to maximise the measured experimental asymmetry, the target electron polarisation $P_{t}$ should be as high as possible. A practical choice for the target is ferromagnetic material for which a polarisation of approximately $8 \%$ can be obtained since at saturation about two of the twenty six electrons of the Fe atom have their spin aligned with the applied magnetic field. Also the target material should provide high magnetic field at saturation which should be reached for low values of the magnetising field. We 
selected the AFK502 alloy $(49 \% \mathrm{Fe}, 49 \% \mathrm{Co}$, and $2 \% \mathrm{~V}$ ) whose saturation induction is 2.35 Tesla; $97 \%$ of the saturation induction is obtained with $H=8000 \mathrm{~A} / \mathrm{m}$. One or several stacked foils of ferromagnetic material with dimensions (length, width, thickness) $L=698 \mathrm{~mm}, l=180 \mathrm{~mm}, t=1.32 \mathrm{~mm}$, and of mass $W=2700.6 \mathrm{~g}$ were installed in the gap of a flat magnetic circuit [18]. To maximise the longitudinal polarisation, the target plane was set at a small angle $\Theta$ with respect to the muon beam direction, limited by the geometry of the foil holder and by the transverse beam dimensions $\left(\Theta=18^{\circ}\right.$ or $\left.25^{\circ}\right)$ (Fig. 5).

The target foil electron polarisation $P_{t}$ is related to the spin contribution $M_{s}$ to the target total magnetisation $M$, and to the electron density of the target material $\rho_{e}$ through $P_{t}=M_{s} /\left(\rho_{e} \mu_{0} \mu_{B}\right)$, where $\mu_{0}$ is the vacuum permittivity, and $\mu_{B}$ the Bohr magneton. The electron density $\rho_{e}$ is related to the material density $\rho=W /$ Llt through $\rho_{e}=N_{A v} \rho \bar{Z} / \bar{A}$, where $\bar{A}$ and $\bar{Z}$ are the average atomic mass and the average atomic number for the target material, respectively.

The ratio of the spin magnetisation to the total magnetisation was deduced from the value of the magnetomechanical ratio $g^{\prime}$ of the material

$$
\frac{M_{s}}{M}=2 \frac{g^{\prime}-1}{g^{\prime}}
$$

A precise determination of the magnetomechanical ratio $g^{\prime}$ of a material is obtained from Einstein-de Haas experiments, in which one observes the mechanical rotation induced on a sample of the material by a change in magnetisation. Unfortunately no $g^{\prime}$ data exist for ternary alloys Fe-Co-V. The most accurate values of $g^{\prime}$ for Fe-Co alloys have been obtained by G. Scott and H. Sturner [19]. For a 50\%Fe 50\%Co alloy $g^{\prime}=1.916 \pm 0.002$. One then derives $M_{s} / M=0.956 \pm 0.002$. A small admixture of vanadium which is a paramagnetic element is not expected to modify substantially this ratio. The value of the uncertainty on $M_{s} / M=0.96 \pm 0.01$ is thought to be a conservative estimate for AFK502.

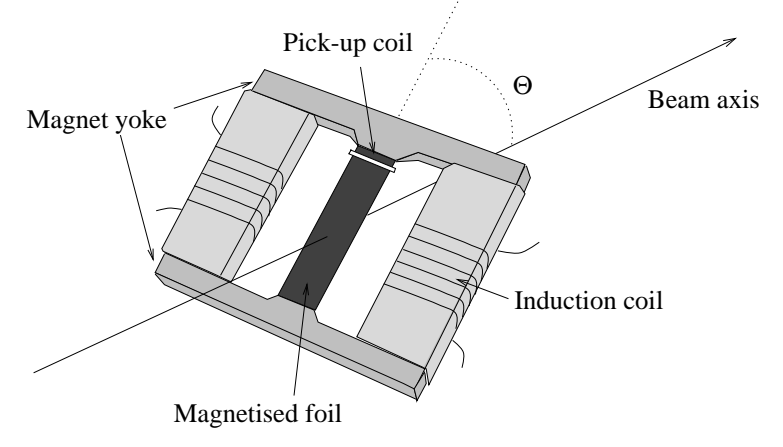

Figure 5: The polarised electron target

The total magnetisation value $M$ for the foil is obtained from the measurement of the change of magnetic flux $\Delta \Phi$ in pick-up coils wrapped around the target, while inverting the current of the magnet coils. The air contribution $\Delta \Phi_{\text {air }}$ to this flux change is determined by performing the same measurement after removing the target foil. Consequently, $\quad M=\left(\Delta \Phi-\Delta \Phi_{\text {air }}\right) /(2 n l t)$, where $\mathrm{n}$ is the number of turns of the pick-up coil. Moving a pick-up coil along the gap the foil magnetisation was checked to be homogeneous within a few permil throughout the area covered by the muon beam. 
is :

Finally, assuming a parallelepipedic shape for the target, the electron polarisation

$$
P_{t}=\frac{M_{s}}{M}\left(\frac{\Delta \Phi-\Delta \Phi_{a i r}}{2 n}\right) /\left(N_{A v} \frac{\bar{Z}}{\bar{A}} \frac{W}{L} \mu_{B} \mu_{0}\right)
$$

For a target angle $\Theta$ we obtain for the longitudinal electron polarisation along the muon beam axis

$$
P_{e}=(0.0834 \pm 0.0010) \cos \Theta
$$

where the error stems mostly from the uncertainty on $g^{\prime}$.

\subsection{The polarimeter spectrometer}

The polarimeter spectrometer is placed downstream of the deep inelastic experiment and takes data concurrently. Parts of the apparatus are common to both the decay and $\mu-e$ scattering polarimeters [9]. Fig. 6 shows the apparatus as set up for $\mu-e$ scattering data taking in 1994. The 1993 set-up differs only in the downstream chamber configuration from that used in 1994. The scattering polarimeter is a two-arm spectrometer allowing for tracking of both incident and scattered particles. It includes hodoscopes, an analysing magnet, and multiwire proportional chambers (MWPC).

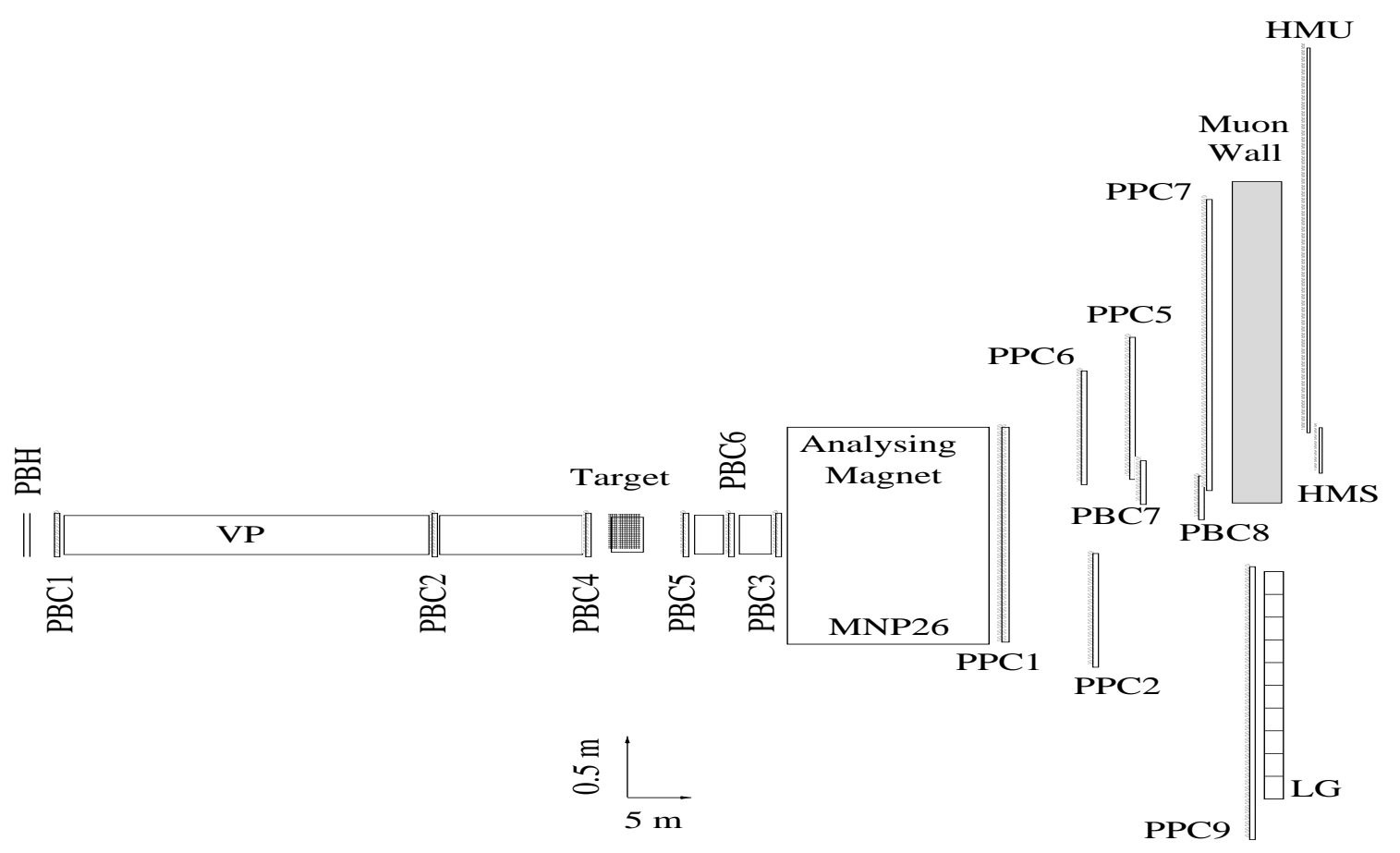

Figure 6: Top view of the experimental set-up for the scattering polarimeter during 1994 data taking.

The polarimeter beam hodoscope (PBH) is composed of two scintillator planes with 32 adjacent elements each. The size of the elements varies so that each element intercepts a similar beam flux. The first plane has its elements oriented vertically (PBHV) and the second one horizontally (PBHH). The total area covered by each plane is $200 \mathrm{~mm}$ by $200 \mathrm{~mm}$. The PBH information is used for timing correlation with other hodoscopes, as part of the trigger, for beam normalisation, and to form roads for the tracking of the particles in the event reconstruction. 
The scattered electron is identified by its shower in a lead glass calorimeter (LG). The lead glass calorimeter is formed from a $3 \times 10$ array of $100 \mathrm{~mm}$ by $100 \mathrm{~mm}, 85 \mathrm{~mm}$ thick lead glass blocks with a radiation length, $X_{0}=24.8 \mathrm{~mm}$. The response of the individual blocks was calibrated using electrons deflected by the analysing magnet. An analog sum of all lead glass signals is also used for the physics trigger.

The scattered muon is identified by a hit in a two part scintillator hodoscope (HMU/S) placed downstream of a $2 \mathrm{~m}$ thick iron wall. The first part (HMS) has 6 adjacent scintillator panels $300 \mathrm{~mm}$ high and of increasing widths $(5,10,15,40,55$, and $80 \mathrm{~mm}$ ) for triggering near the beam. The second part (HMU) is composed of 22 adjacent scintillator panels $300 \mathrm{~mm}$ high and $75 \mathrm{~mm}$ wide. The first HMU element and the last HMS element have an overlap of roughly $30 \mathrm{~mm}$ to avoid any gap in the acceptance. A logical sum of all elements is used for the physics trigger.

For all the elements of the PBH, LG, and HMU/S, the charge of the signal and the timing relative to the trigger are measured for each event, and the counting rates are permanently monitored.

The analysing magnet (MNP26) is $6 \mathrm{~m}$ long and has a gap $500 \mathrm{~mm}$ in width by $140 \mathrm{~mm}$ in height. It is operated at $\int B d l$ values 8.5 and 11.7 T.m for which the field has been mapped. The analysis had to account for the fact that low momentum particles could be significantly influenced by the fringe field. The stability of the field is continuously monitored with Hall and NMR probes. The BMS was calibrated with respect to the MNP26 to an accuracy of $2 \times 10^{-3}$ by deflecting the muon beam through the muon polarimeter and measuring the same muons through both apparatus.

A set of MWPC's provides the tracking information. The tracking volume is split into four telescopes : tracking upstream of the target, tracking downstream of the target, tracking of negative particles downstream of the magnet $\left(e^{-}\right)$, and tracking of positive particles downstream of the magnet $\left(\mu^{+}\right)$. The first and second telescopes are formed from three PBC chambers each designed to operate in the beam. In each of these telescopes vacuum tubes are placed between the chambers in order to reduce secondary interactions. All of the PBC's use the RMH [20] readout system developed at CERN .

As shown in Fig. 6 there are 6 chambers for tracking the scattered $\mu^{+}$and 3 chambers for tracking the scattered $e^{-}$. PPC7 is read out with RMH electronics while the other PPC's are read out with PCOS. The parameters of all the chambers are listed in Table 3.3.

\begin{tabular}{|c|l|c|c|}
\hline chamber & wire angles & pitch $(\mathrm{mm})$ & active area $\left(\mathrm{mm}^{2}\right)$ \\
\hline PBC1-8 & $0^{\circ}, 90^{\circ}, 45^{\circ},-45^{\circ}$ & 1 & $192 \times 192$ \\
\hline PPC1 & $0^{\circ}, 0^{\circ}, 36.9^{\circ},-36.9^{\circ}$ & 1 & $960 \times 240$ \\
PPC2 & $0^{\circ}, 90^{\circ}, 28^{\circ}$ & 2 & $500 \times 300$ \\
PPC5 & $0^{\circ}, 16.6^{\circ},-15.5^{\circ}$ & 2 & $600 \times 400$ \\
PPC6 & $0.2^{\circ}, 90^{\circ},-27.9^{\circ}$ & 2 & $500 \times 300$ \\
PPC7 & $0^{\circ}, 0^{\circ}, 36.9^{\circ},-36.9^{\circ}$ & 2 & $2000 \times 400$ \\
PPC9 & $0^{\circ}, 0^{\circ}, 28.1^{\circ},-28.2^{\circ}$ & 2 & $1300 \times 400$ \\
\hline
\end{tabular}

Table 1: Polarimeter proportional chamber parameters. Angles are given relative to positive vertical axis 


\subsection{Trigger and data acquisition}

Three types of triggers are recorded : beam, physics, and normalisation. The beam triggers are used to monitor beam conditions, check target field polarity through curvature of the muon track, and provide input tracks for the Monte Carlo. They are formed by requiring at least one hit in each $\mathrm{PBH}$ plane. The physics trigger is a beam trigger in coincidence with a hit in the HMU/S hodoscope and a shower in the LG with a deposition of at least $20 \mathrm{GeV}$. This trigger condition together with the experimental set-up define the $y$ acceptance ranging from $y=0.10$ to $y=0.90$. The normalisation trigger [21], used to measure the effective beam flux $\Phi$, is produced by the coincidence between a beam trigger and a signal randomly distributed in time. The random signal is obtained from the coincidence of pulses from two phototubes viewing two scintillators sandwiching a ${ }^{241} \mathrm{Am}$ source. This system is located far away from the muon beam line in order to avoid detection of particles originating in the beam line.

All triggers are combined in a fan-in module whose output signal starts the data acquisition; they are therefore affected in the same way by data acquisition dead-time which is typically $35 \%$. Since the number of $\mu$-e scattering events and of normalisation events play a symmetrical role in the determination of the asymmetry, they should be about the same in order to optimise the overall statistical error. Typical trigger rates are 500 physics triggers per burst, 200 normalisation triggers per burst, and 70 beam triggers per burst.

The detector readout comprises three CAMAC branches which are read out by VME-based branch controllers run by a FIC module. The branches correspond roughly to beam chambers, PCOS chambers, and ADC/TDC readout. The BMS information is transferred to a CAMAC memory module. The ADC/TDC branch is read out directly by VME high speed memory modules. After readout and event building, the events are sent to a SUN workstation for online monitoring and to a VAX cluster for writing to exabyte $8 \mathrm{~mm}$ tapes.

\section{MEASUREMENTS AND ANALYSIS}

\subsection{Experimental procedure}

The beam polarisation is deduced from the experimental asymmetry in $\mu-e$ scattering when the target polarisation is inverted by reversing the magnetising field. This field affects the trajectories of the reaction particles in a different way depending on the polarisation of the target, thereby inducing a false asymmetry. Because of the azimuthal symmetry of the scattering process around the beam direction, the false asymmetry is induced only by the vertical component of the magnetic field $B_{v}$. In order to correct for this false asymmetry, we performed measurements for the two target polarisation orientations at two target inclination angles with respect to the horizontal plane : $\Theta$, and $\pi-\Theta$ (see Fig. 7). In a given $y$ bin, the measured yields for antiparallel and parallel relative beam and target spin orientations, and for the two target inclination angles are :

$$
\begin{aligned}
N_{a}(\Theta) & =\Phi_{a}(\Theta) n \sigma_{0} a_{-}(\Theta)\left(1+P_{\mu} P_{e} A\right) \\
N_{p}(\Theta) & =\Phi_{p}(\Theta) n \sigma_{0} a_{+}(\Theta)\left(1-P_{\mu} P_{e} A\right) \\
N_{a}(\pi-\Theta) & =\Phi_{a}(\pi-\Theta) n \sigma_{0} a_{+}(\pi-\Theta)\left(1+P_{\mu} P_{e} A\right) \\
N_{p}(\pi-\Theta) & =\Phi_{p}(\pi-\Theta) n \sigma_{0} a_{-}(\pi-\Theta)\left(1-P_{\mu} P_{e} A\right)
\end{aligned}
$$

where $\Phi$ is the muon flux, $\mathrm{n}$ is the number of electrons, and $\sigma_{0}$ the unpolarised crosssection. The apparatus acceptance (which includes apparatus efficiencies in addition to 


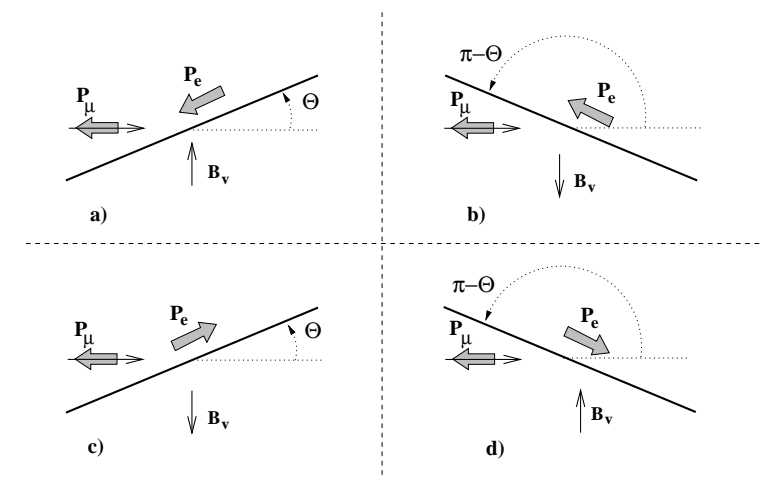

Figure 7: The four configurations measured to cancel the false asymmetry induced by the vertical field component $B_{v}$ of the target magnetic circuit.

geometrical acceptance) is $a_{+}\left(a_{-}\right)$for a positive (negative) vertical field component. Defining the experimental asymmetry $\Delta=\left(N_{a} / \Phi_{a}-N_{p} / \Phi_{p}\right) /\left(N_{a} / \Phi_{a}+N_{p} / \Phi_{p}\right)$, one can extract the beam polarisation

$$
P_{\mu}=\frac{1}{P_{e} A} \frac{1+\Delta(\Theta) \Delta(\pi-\Theta)-\sqrt{\left(1-\Delta(\Theta)^{2}\right)\left(1-\Delta(\pi-\Theta)^{2}\right)}}{\Delta(\Theta)+\Delta(\pi-\Theta)}
$$

which when all $\Delta \mathrm{s}$ are small is,

$$
P_{\mu} \simeq \frac{1}{P_{e} A} \frac{\Delta(\Theta)+\Delta(\pi-\Theta)}{2}
$$

This relation is valid only if the ratio $a_{+} / a_{-}$remains constant throughout the set of measurements. In practice, the target magnetisation was reversed on a pulse to pulse basis whereas the target angle was changed from $\Theta$ to $\pi-\Theta$ every two hours. The acceptance variations with time which reached up to $\pm 10 \%$ were mostly due to chamber efficiency variations.

Data were analysed in $y$ bins. This provided an overall check of the measurement, since the variation of the experimental asymmetry as a function of $y$ should be equal to that of the theoretical asymmetry up to the constant proportionality factor $P_{\mu} P_{e}$.

The $y$ value for each event was defined as the relative muon energy loss (involving only the muon energies measured by the BMS and the polarimeter spectrometer), rather than the ratio of the recoil electron energy to the incoming muon energy. This minimised the effect of radiative energy loss on the determination of the $y$ value at the vertex of the reaction.

\subsection{Tracking}

Linear track segments were computed using proportional chamber information in each of the four telescopes [22][23]. The hit hodoscope counters (PBH on the incident track, HMU on the scattered muon track, and LG on the recoil electron track) were used as filters in the wire selection for the proportional chambers.

\subsubsection{Incident and scattered track reconstruction}

First, the tracks upstream of the target were reconstructed. A minimum of 7 hit planes out of a total of 12 was required. 
In order to reconstruct track segments between the target and the analysing magnet, roads were defined using the intersection of reconstructed incident tracks with the target, and points reconstructed in the last chamber upstream of the analysing magnet. For all the combination of pairs of points (one in the target, and one in the last chamber) a road was defined in which tracks were searched with a minimum of 8 hit planes. Tracks corresponding to a scattering angle less than $1 \mathrm{mrad}$ were discarded both upstream and downstream of the target.

\subsubsection{Vertex reconstruction}

The vertex search was started by looking for the point with a minimum sum of squared distances to all the scattered tracks downstream of the target. The track with maximum distance was discarded whenever its distance was larger than $1 \mathrm{~mm}$, and a new vertex search was performed. Only events with two remaining tracks were kept.

The matching between the incident track and the two remaining tracks was done taking each incident track one by one and calculating a vertex. The best incident track, corresponding to the lowest sum of squared distances for this vertex was kept. Events with this sum larger than $3 \mathrm{~mm}^{2}$ were discarded. Finally the track parameters were recalculated imposing the vertex as a common point. The resulting angular resolution on scattering angles was better than $0.1 \mathrm{mrad}$.

\subsubsection{Track identification and momentum determination}

The reconstruction downstream of the magnet was performed in the same way for the muon and for the electron. For each track the intersection with the vertical plane at the center of the analysing magnet was computed. Roads for the wire selection were determined using this point and the hit elements of hodoscopes HMU or LG.

The momentum was determined with the upstream and the downstream tracks using a parametrisation of the magnet transfer function deduced from the measured field map. The resulting relative momentum resolution was estimated to be better than $1 \%$.

In order to identify correctly the scattered muon and the recoil electron, combinations using both upstream tracks were tried, and the $\chi^{2}$ of the fits were compared. For $3.5 \%$ of the events, the best muon and the best electron reconstructed trajectories corresponded to the same upstream track; they were rejected.

\subsection{Analysis}

\subsubsection{Data taken and data selection}

Data were taken using the $\mu-e$ scattering polarimeter during the 1993 and 1994 SMC running periods as summarised in Table 2. Polarised targets (AFK502) and unpolarised targets (polyethylene and copper) of various thicknesses were used in both 1993 and 1994. In 1993 the polarised target consisted of 2 foils of AFK502 tilted at an angle of $25^{\circ}$ while in 1994 targets of 2 foils and 4 foils were used at an angle of $18^{\circ}$. As listed in Table 2, these three configurations correspond to three effective target thicknesses along the beam direction $\left(0.37 X_{0}, 0.50 X_{0}\right.$, and $\left.0.95 X_{0}\right)$. There were minor differences in the analysis cuts and in the background correction procedure for the three target thicknesses. This Section describes in detail the analysis of the data collected with the $0.50 X_{0}$ target.

The final set of data was selected by checking beam and apparatus status. For each burst the same minimal beam intensity was required as in the deep inelastic SMC analysis. Each run contained data taken at one target angle over a period of approximately 2 hours. The edge $y$ bins were discarded to avoid any biases from regions of low acceptance and 


\begin{tabular}{|c|c|c|l|c|c|}
\hline Year & Beam & $\begin{array}{c}E_{\text {beam }} \\
(\mathrm{GeV})\end{array}$ & $\begin{array}{l}\text { target } \\
\text { material }\end{array}$ & $\begin{array}{c}\text { effective target } \\
\text { thickness }\left(X_{0}\right)\end{array}$ & $\begin{array}{c}\text { number of } \\
\text { physics triggers }\left(10^{6}\right)\end{array}$ \\
\hline 1993 & $\mu^{+}$ & 100 & AFK502 & 0.37 & 8. \\
& $\mu^{+}$ & 190 & AFK502 & 0.37 & 13. \\
& $\mu^{+}$ & 190 & $\left(\mathrm{CH}_{2}\right)_{\mathrm{n}}$ & 0.37 & 10. \\
\hline 1994 & $\mu^{+}$ & 190 & AFK502 & 0.50 & 15. \\
& $\mu^{-}$ & 190 & AFK502 & 0.50 & 0.4 \\
& $\mu^{+}$ & 190 & $\left(\mathrm{CH}_{2}\right)_{\mathrm{n}}$ & 0.50 & 9. \\
& $\mu^{+}$ & 190 & AFK502 & 0.95 & 10. \\
& $\mu^{+}$ & 190 & Cu & 0.95 & 1.1 \\
& $\mu^{+}$ & 190 & None & 0.00 & \\
\hline
\end{tabular}

Table 2: Summary of $\mu-e$ scattering data taking

large target field effects. Periods were defined as sets of runs for which the status of the apparatus was stable. All the runs of a period were combined.

Approximately $10 \%$ of the physics triggers were reconstructed and passed cuts for use in the final data set.

\subsubsection{Asymmetry dilution effects}

The asymmetry was diluted by scattering events in the air around the target. The size of this effect was studied with a Monte Carlo simulation and using data from runs without target. This gave a relative correction to the asymmetry of order $1 \%$ depending on target thickness with an error of $0.1 \%$. The stability of the correction was checked by varying the vertex cut. The final vertex cut was set at a distance of $\pm 123 \mathrm{~mm}$ of the target plane.
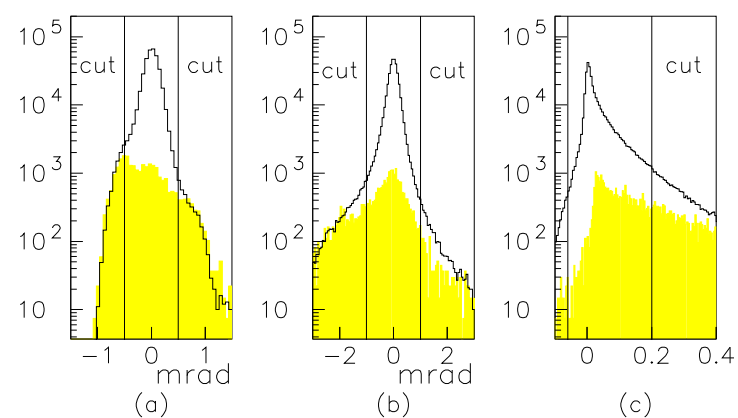

Figure 8: Spectra of (a) $\theta_{\mu}^{\text {measured }}-\theta_{\mu}^{\text {calc }}(y)$, (b) $\theta_{e}^{\text {measured }}-\theta_{e}^{\text {calc }}(y)$, and $(c)\left(E_{\mu}-E_{\mu}^{\prime}-E_{e}^{\prime}\right) / E_{\mu}$ for $\mu^{+}$data. The shaded areas are the same quantities from the $\mu^{-}$data. The vertical lines indicate the cuts applied in the analysis.

The asymmetry was also diluted by muon bremsstrahlung and direct pair production events in the target. Several kinematic cuts were applied to reject these events. These included cuts on the difference between the measured value of the muon scattering angle and the value calculated from the measured $y$, on the difference between measured and calculated electron scattering angle, and on the fractional missing energy, $\left(E_{\mu}-E_{\mu}^{\prime}-E_{e}^{\prime}\right) / E_{\mu}$. 
These cuts allowed a discrimination between bremsstrahlung and elastic scattering events as described in Sec. 2 and Fig. 4 . For the $0.50 X_{0}$ target the cut values were :

$$
\begin{aligned}
& \left|\theta_{\mu}^{\text {measured }}-\theta_{\mu}^{\text {calc }}(y)\right| \leq 0.5 \mathrm{mrad} \\
& \left|\theta_{e}^{\text {measured }}-\theta_{e}^{\text {calc }}(y)\right| \leq 1.0 \mathrm{mrad}, \\
& \text { and }-0.06 \leq\left(E_{\mu}-E_{\mu}^{\prime}-E_{e}^{\prime}\right) / E_{\mu} \leq 0.2 .
\end{aligned}
$$

They are illustrated in Fig. 8. The angular cuts and the upper cut on missing energy rejected radiative background. The lower cut on missing energy rejected incorrectly reconstructed events containing tracks from non-interacting beam muons.

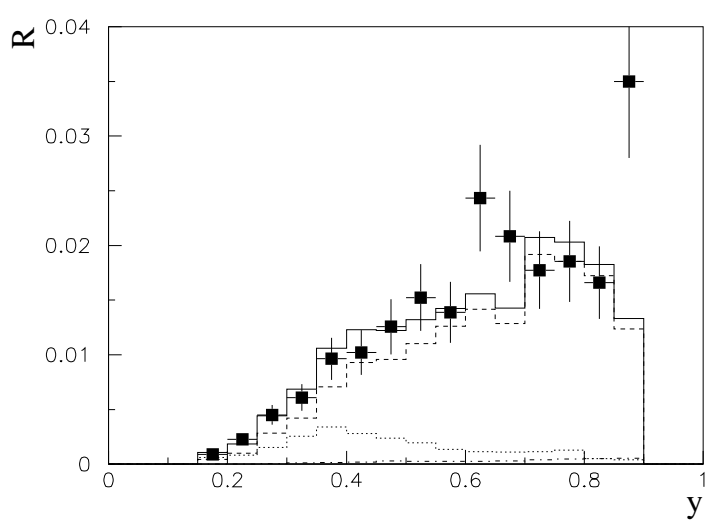

Figure 9: Relative background level after cuts, $R$, for $19940.50 X_{0}$ measurement. The solid squares are the results obtained from the ratio of $\mu^{-}$to $\mu^{+}$data. The curves are results from Monte Carlo. The solid curve represents the sum of all processes, the dashed curve the contribution from muon bremsstrahlung conversion, the dotted curve that from showers from $\mu^{-}-e^{-}$scatters, and the dash-dotted curve that from direct pairs.

The same cuts were applied to the $\mu^{-}$data. Fig. 9 shows the background ratio $r_{B G}(y)$. The background was treated as a dilution of the measured polarisation which was corrected by multiplying by $1 /\left(1-r_{B G}(y)\right)$. Since $\mu^{-}$data were taken only with the $0.50 X_{0}$ target thickness they were used to correct the $0.50 X_{0}$ data and extrapolated toward zero for the $0.37 X_{0}$ data.

The background level was also estimated using a Monte Carlo simulation. A comparison with the $\mu^{-}$result is shown in Fig. 9. This simulation was used to correct the $0.95 X_{0}$ result and as a crosscheck for the $0.37 X_{0}$ and $0.50 X_{0}$ results.

\subsubsection{Polarisation calculation}

For each period the beam polarisation was calculated for each $y$ bin through Eq. 15 . In Fig. 10 we present for the three measurements at $190 \mathrm{GeV}$, the combined values of the beam polarisation for all periods. The constancy of the polarisation value as a function of $y$ is an overall check of the consistency of our measurement. One should however note that the represented error bars in Fig. 10 do not take into account correctly the correlations induced by the normalisation which is common to all bins.

In the final analysis, for each period the beam polarisation was extracted by fitting the beam flux to the number of normalisation triggers, and for each $y$ bin the number of $\mu-e$ events to the expressions (11) to (14) with the assumption that the ratio of 


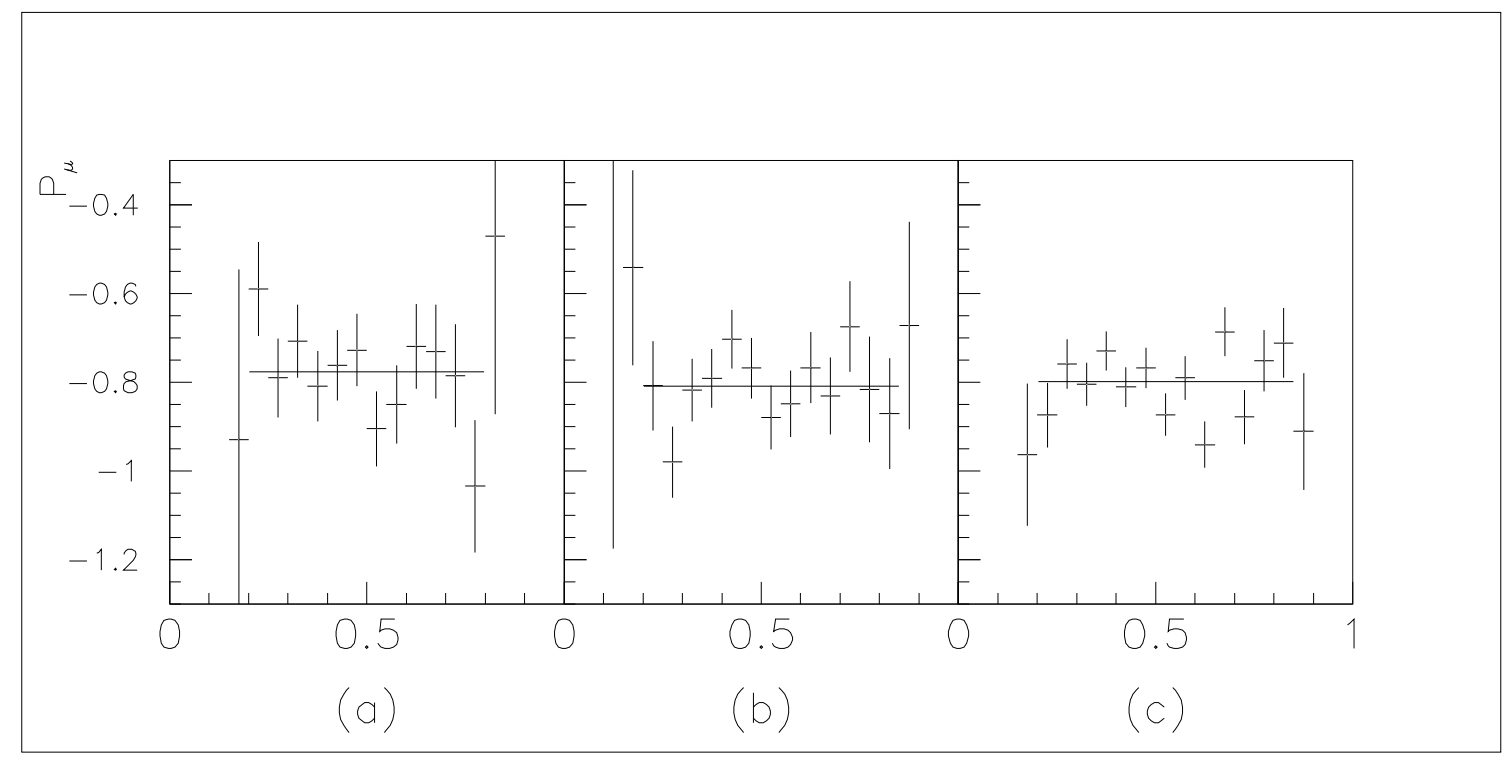

Figure 10: Polarisation at $190 \mathrm{GeV}$ in bins of y for (a) 1993 measurement with 2 foils, (b) 1994 measurement with 2 foils, and (c) 1994 measurement with 4 foils. The solid lines indicate the $y$ bins used in the weighted mean.

acceptances for + and - field was the same for both target angles. The $\chi^{2}$ was minimised using MINUIT. Altogether there were 47 parameters (beam polarisation, 4 beam flux values, and 28 acceptance values corresponding to $a_{-}(\Theta)$ and $a_{-}(\pi-\Theta)$ and 14 acceptance ratios $a_{+} / a_{-}$for the $14 y$ bins covering the range from $y=0.15$ to $y=0.85$ ), and 60 experimental counting values. At $190 \mathrm{GeV}$ nominal beam energy the average $\chi^{2}$ (for the 13 degrees of freedom) was $12.3,13.1$, and 11.7 respectively for the $1993\left(0.37 X_{0}\right)$, $1994\left(0.50 X_{0}\right)$, and the $1994\left(0.95 X_{0}\right)$ measurements.

For each measurement the polarisation value and its statistical error were calculated by combining the beam polarisation values for the different periods.

\subsubsection{Systematic studies}

The errors are summarised in Table 3 for all three measurements at $190 \mathrm{GeV}$ nominal beam energy. Correlated errors are listed in a separate column. The errors are dominated by counting statistics. The next largest error is due to the target polarisation. The various cuts on angles and missing energy were set in a position where the result was stable. The cut sensitivity was estimated by varying around this cut. The angular cuts were placed at $4 \sigma$ of the peak width and varied between $2 \sigma$ and $6 \sigma$. The missing energy cut was placed at 0.2 and varied between 0.1 and 0.4 . The fluctuations in the stable region were within the error quoted for cut sensitivity. The errors due to momentum resolution and alignment were added in quadrature and labeled calibrations.

Possible residual false asymmetries due to acceptance variation with time were studied in two different ways. First, data were taken with non polarised targets. The results were all consistent with zero: $-0.022 \pm 0.026\left(0.37 X_{0}\left(C_{2}\right)_{n}\right), 0.006 \pm 0.029$ $\left(0.50 X_{0}\left(\mathrm{CH}_{2}\right)_{n}\right),-0.033 \pm 0.026\left(0.95 \mathrm{X}_{0} \mathrm{Cu}\right)$.

Residual false asymmetries were also tested by combining runs with the polarised target far away in time. Comparing the resulting asymmetries to those obtained with data adjacent in time provides an overestimate of the residual false asymmetry. It was found 


\begin{tabular}{|l|c|c|c|c|}
\hline Error source & 1993 & $1994\left(0.50 X_{0}\right)$ & $1994\left(0.95 X_{0}\right)$ & common \\
\hline Statistics & 0.031 & 0.026 & 0.013 & \\
$P_{e}$ & - & - & - & 0.009 \\
Cut sensitivity & 0.005 & 0.005 & 0.006 & \\
Residual false asymmetry & 0.005 & 0.005 & 0.004 & \\
Calibrations & - & - & - & 0.004 \\
Background & - & - & - & 0.003 \\
Radiative corrections & - & - & - & 0.002 \\
Transverse asymmetry & - & - & - & 0.001 \\
Levchuk effect & - & - & - & 0.001 \\
Air dilution & - & - & & 0.001 \\
\hline
\end{tabular}

Table 3: Errors for $\mu-e$ measurements of the beam polarisation in 1993 and 1994

compatible with zero within the normalisation error for each of the three measurements. These two estimates were used to assign the error ascribed to the residual false asymmetry.

The level of agreement between the background measured with the $\mu^{-}$beam and estimated from the Monte Carlo result was used to estimate the background correction systematics. The $\mu^{-}$data were used to correct the $0.50 X_{0}$ result, scaled by the target thickness to correct the $0.37 X_{0}$ result, and used as a crosscheck of the correction for the $0.95 X_{0}$ result. For this reason it is treated as a correlated error.

The radiative corrections were calculated using the final set of cuts. The ratio of corrected asymmetry to Born asymmetry is shown in Fig. 3. Since the effect is small compared to the final error, it was counted as an error on the Born asymmetry rather than as a correction.

The effect of the transverse muon beam polarisation on the experimental asymmetry has been estimated using the muon spin precession angle in the beam transport system and the value of the $a_{z x}$ theoretical asymmetry. The resulting maximum asymmetry is about 0.002 . However, because of the azimuthal symmetry of the acceptance, the effect almost cancels out.

We have examined the incidence of the Levchuk effect [24] on the asymmetry value. Because of the smearing of the kinematics for the scattering process from non polarised inner shell target electrons with high average momentum (up to $\approx 100 \mathrm{keV} / \mathrm{c}$ ) as compared to the case of the scattering off the polarised $3 \mathrm{~d}$ shell electrons almost at rest, angular cuts operated during the analysis produce a bias leading to an overestimation of the beam polarisation. The effect is expected to be small due to the fact that the smearing is less than the angular resolution of the reconstruction. Using crude approximations for the momentum distribution of the target electrons [25] in a Monte Carlo simulation, we estimated the corresponding contribution to the extracted beam polarisation value to be less than 0.001 .

The $190 \mathrm{GeV}$ data for both the 1993 and the $19940.50 X_{0}$ target were also analysed with another analysis chain which used an alternate reconstruction program with a different tracking algorithm, thus providing an independent result using the same input data [26]. This program differed primarily in its use of points reconstructed in the chambers instead of individual planes for the tracking upstream of the analysing magnet. For both years the results using both methods were compatible within statistical error. 


\subsection{5 $100 \mathrm{GeV}$ analysis}

During 1993, data were also taken at a beam energy of $100 \mathrm{GeV}$. These data were analysed with the alternate reconstruction method. For this measurement there were no $\mu^{-}$data to use for the background correction. The size of the tails of the $\left|\theta_{\mu}^{\text {measured }}-\theta_{\mu}^{\text {calc }}(y)\right|$ spectra are directly related to the background level. A Monte Carlo simulation was used along with these tails to calculate the correction. The systematic error was somewhat larger due to increased uncertainty on the background correction.

\subsection{Results and conclusion}

The results of the $P_{\mu}$ measurements at 100 and $190 \mathrm{GeV} / \mathrm{c}$ in 1993 and 1994 are listed in Table 4 with the error separated into statistical, uncorrelated systematic, and correlated systematic components. The average beam energy for these measurements is given

\begin{tabular}{|l|c|c|c|c|c|}
\hline Measurement & $\left\langle E_{\text {beam }}\right\rangle(\mathrm{GeV})$ & $P_{\mu}$ & stat. & $\begin{array}{c}\text { uncorr. } \\
\text { syst. }\end{array}$ & $\begin{array}{c}\text { corr. } \\
\text { syst. }\end{array}$ \\
\hline \hline $1993\left(0.37 X_{0}\right)$ & 99.4 & -0.804 & 0.032 & 0.021 & 0.011 \\
\hline \hline $1993\left(0.37 X_{0}\right)$ & 187.4 & -0.763 & 0.031 & 0.007 & 0.011 \\
$1994\left(0.50 X_{0}\right)$ & 187.4 & -0.807 & 0.026 & 0.007 & \\
$1994\left(0.95 X_{0}\right)$ & 187.4 & -0.800 & 0.013 & 0.007 & \\
\hline Combined & 187.4 & -0.797 & 0.011 & 0.004 & 0.011 \\
\hline
\end{tabular}

Table 4: Summary of $\mu-e$ scattering results for $P_{\mu}$ showing statistical, uncorrelated systematic, and correlated systematic errors

at the SMC polarised target. The weighted average was calculated using the statistical and uncorrelated systematic errors to compute the weights.

The beam polarisation was also measured by fitting the energy spectrum of the positrons from muon decay (see Addendum and Refs. [9, 10]). Table 5 shows the results of the polarisation measurements at the two beam energies. Here statistical and systematic errors have been added in quadrature. For the $190 \mathrm{GeV}$ beam, the two methods give compatible results, so a final measured polarisation $P_{\mu}$ was calculated by taking the weighted mean. The final error contains an additional contribution of 0.005 , estimated using the beam line Monte Carlo, to take into account changes in the hadron slit aperture.

\begin{tabular}{||c|c|c||c|}
\hline$<E_{\text {beam }}>(\mathrm{GeV})$ & $P_{\mu}^{\mu-e}$ & $P_{\mu}^{\text {decay }}$ & $P_{\mu}$ \\
\hline 99.4 & $-0.80 \pm 0.04$ & & $-0.80 \pm 0.04$ \\
\hline 187.4 & $-0.797 \pm 0.016$ & $-0.806 \pm 0.029[10]$ & $-0.799 \pm 0.015$ \\
\hline
\end{tabular}

Table 5: Summary of the beam polarisation measurements.

The measured polarisation values can be compared to those calculated using a Monte Carlo simulation [15] which includes full description of the beam line and of the hadron decay along the decay channel. This gave a result for $P_{\mu}$ of $-0.83 \pm 0.04$ at $99.4 \mathrm{GeV}$ and $-0.79 \pm 0.04$ at $187.4 \mathrm{GeV}$ [27] in good agreement with the measured values.

In conclusion, the $\mu-e$ scattering method has been shown to provide a high accuracy measurement of the muon beam polarisation with significant advantages over measurements based on the study of the $\mu$ decay : 
- the physical process is clearly identified

- the background is directly measured

- because we perform an asymmetry measurement, uncertainties on the detector efficiency cancel out, and therefore no Monte-Carlo simulation is needed in order to derive the beam polarisation value

Data were taken at two beam energies and in several configurations. The final results are in agreement both with the measurement using the muon decay spectrum and with calculations using the beamline simulation. The error on the final measured polarisation is below $2 \%$. This achievement contributes to the reduction of the systematic error in the SMC spin asymmetry measurements.

We dedicate this paper to the memory of our beloved secretary Elizabeth Cumiskey who served the CERN muon collaborations EMC, NMC, and SMC from 1983 to 1994. We thank CERN, our host laboratory for providing us the support needed for the successful completion of our experiment. The technical assistance given by J.-M. Demolis in setting up, operating, and maintaining our detectors is gratefully acknowledged. We wish to express our gratitude to Dr D. Bardin for helping us in the understanding of the radiative corrections aspects of our measurement.

\section{Addendum: Measurement using the decay positron energy spectrum}

The polarisation was also measured, for the beam energy of $190 \mathrm{GeV}$, by analysing the energy spectrum of the positron from $\mu^{+} \rightarrow e^{+} \nu_{e} \bar{\nu}_{\mu}$ decay. Measuring the parent (beam) muon energy $E_{\mu}$ and the energy $E_{e^{+}}$of the outgoing positron for each event, one can study the distribution of the variable $y=E_{e^{+}} / E_{\mu}$. The shape of this distribution depends on the muon polarisation and can be computed in QED with a high accuracy.

This alternate method is relatively "fast", because the number of events necessary to calculate $P_{\mu}$ with the statistical accuracy of $\sim 1 \%$ can be collected in one week of data taking. However, the "decay" method relies on the Monte Carlo acceptance calculations and therefore requires an accurate description of all detectors, chamber efficiencies and physical subprocesses [22].

A large part of the apparatus [9] is common with that of the $\mu-e$ scattering asymmetry measurement (Fig. 6). A lead foil located before PBH allows it to be used as a shower veto to reject positron from upstream decay. The decay area extends between the $\mathrm{PBH}$ and the analysing magnet and includes only the three chambers, PBC1, PBC2 and PBC3. The magnet field is reversed so that decay positrons are deflected into the LG telescope which includes PPC2, PPC9 and another similar chamber in-between.

The analysis was refined in many ways [10] with respect to Ref. [9]. A similar tracking as in the $\mu-e$ asymmetry measurement was used and the alignment was improved. Monte Carlo events were weighted to take into account the muon energy dependence of the decay probability. An underestimate of the decay angle was obtained, assuming that the decay occurred in PBC2. Events for which this estimate was larger than the kinematical maximum were rejected. A new selection procedure was designed in order to eliminate background events produced by muons which do not decay but get deflected enough in the magnet to reach LG and generate a decay trigger. It rejects events with a downstream track close to the beam edge of LG, an upstream track on the LG side of PBC3 and an estimated decay angle compatible with zero $\left(\theta_{\text {decay }}<0.15 \mathrm{mrad}\right)$. Due to this new cut, the positron identification cut could be tightened to about $\left|P_{e}-E_{\mathrm{LG}}\right|<24 \mathrm{GeV}$. Also, 


\begin{tabular}{|l|l|}
\hline Error source & \\
\hline BMS calibration $\left(p_{e} / p_{\mu}\right)$ & 0.014 \\
Background & 0.012 \\
\hline Radiative corrections & 0.010 \\
Alignment & 0.008 \\
Beam contamination by $e^{+}$ & 0.005 \\
BMS efficiency fluctuations & 0.005 \\
Geometrical acceptance & 0.010 \\
Total & 0.026 \\
\hline
\end{tabular}

Table 6: Systematic errors for the measurement of the polarisation from the decay positron energy spectrum.

more cuts were introduced : a fiducial cut at the level of PPC2 and a cut on low positron momentum $\left(P_{e}>52 \mathrm{GeV}\right)$ which improved the background rejection.

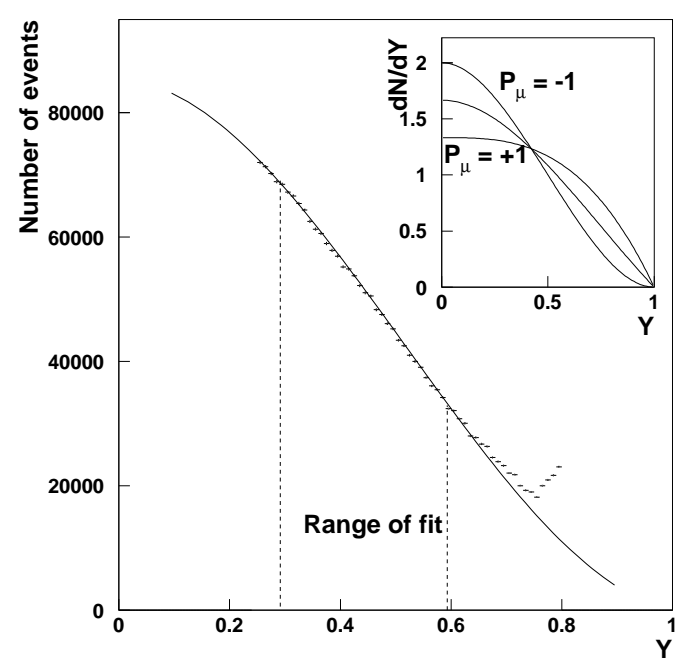

Figure 11: Experimental positron spectrum from muon decay, $\mu^{+} \rightarrow e^{+} \nu_{e} \bar{\nu}_{\mu}$, after acceptance correction, together with the Michel spectrum for $P_{\mu}=-0.806$. The shape of the Michel spectra for $P_{\mu}=-1,0$ and 1 is shown in the insert in the upper right corner.

Fitting over the range [0.26-0.58] for $y=E_{e^{+}} / E_{\mu}$ as illustrated in Fig. 11, we get $P_{\mu}=-0.806 \pm 0.013 \pm 0.026$ for an average energy of $187.4 \mathrm{GeV}$, where the first error is statistical and the second one is systematic. The main sources of systematic errors are listed in Table 6. 


\section{References}

[1] J. Ashman et al., Nucl. Phys. B328 (1989) 1.

[2] B. Wagner et al.,Nucl. Instr. and Meth. A294 (1990)541.

[3] J. Arrington et al., Nucl. Instr. and Meth. A311 (1992)39.

[4] D. Barber et al., Nucl. Instr. and Meth. A329 (1993) 79.

[5] M. Woods, et al., Proc. SPIN 96 Conference, World Scientific, Singapore, 1996, p. 843.

[6] G. Backenstoss et al., Phys. Rev. Lett. 6 (1961) 415.

[7] SMC Collaboration, B. Adeva et al., Phys. Lett. B412 (1997) 414, and Phys. Rev. D58 (1998) 112001.

[8] SMC Collaboration, D. Adams et al., Phys. Lett. B396 (1997) 338.

[9] SMC Collaboration, B. Adeva et al., Nucl. Instr. and Meth. A343 (1994) 363.

[10] J. Cranshaw et al., ,SMC note 97/17, 1997 (unpublished).

[11] J. Cranshaw, The SMC Muon-Electron Elastic Scattering Polarimeter, PhD thesis, Rice University,(1995).

[12] E. Burtin, Mesure de la polarisation d'un faisceau de muons de $190 \mathrm{GeV}$ par diffusion sur une cible d'électrons polarisés, PhD thesis, DAPNIA/SPhN-96-01T (1996).

[13] D. Bardin and L. Kalinovskaya, DESY-97-230, (1997).

[14] P. Schüler, A Muon Polarimeter Based on Elastic Muon-Electron Scattering, Proc. 8th Int. Symp. on High Energy Spin Physics, Mineapolis, 1988.

[15] N. Doble et al., Nucl. Instr. and Meth. A328 (1994) 351.

[16] SMC Collaboration, D. Adams et al., Phys. Rev. D56 (1997) 5330.

[17] V. Bargmann, L. Michel, and V.L. Telegdi, Phys. Rev. Lett. 2 (1959) 435.

[18] N. de Botton, A. Dael, and J. Martino, IEEE Trans. Magnetics 30 (1994) 2447.

[19] G. Scott, and H. Sturner, Phys. Rev. 184 (1969) 490.

[20] J. B. Lindsay et al., Nucl. Instr. and Meth. 156 (1978) 379.

[21] R.P. Mount, Nucl. Instr. and Meth. 187 (1981) 401.

[22] A. de Lesquen et. al., The SMC Beam Polarisation Measurement, in Proc. of Deep Inelastic Scattering and QCD Conf., Chicago (1997) 829.

[23] E. Burtin et al., SMC note 98/01, 1998 (unpublished).

[24] L. G. Levchuk, Nucl. Instr. and Meth. A345 (1994) 496.

[25] N. de Botton, SMC note 95/08, 1995 (unpublished).

[26] F. Simeoni, Misura della polarizzazione del fascio di muoni dell'esperimento SMC, PhD thesis, Trieste (1997) (unpublished).

[27] L. Gatignon, private communication. 